\title{
DEGENERATE FORMS OF MAXWELL'S EQUATIONS
}

\author{
A. L. CAREY AND K. MCNAMARA
}

(Received 7 July 1988; revised 18 May 1989)

\begin{abstract}
This paper studies degenerate forms of Maxwell's equations which arise from approximations suggested by geophysical modelling problems. The approximations reduce Maxwell's equations to degenerate elliptic/parabolic ones. Here we consider the questions of existence, uniqueness and regularity of solutions for these equations and address the problem of showing that the solutions of the degenerate equations do approximate those of the genuine Maxwell equations.
\end{abstract}

\section{Introduction}

We begin by recalling Maxwell's equations:

$$
\begin{aligned}
\nabla \times E & =-\partial_{t} B \\
\nabla \times H & =\partial_{t} D+J \\
\nabla \cdot B & =0 \\
\nabla \cdot D & =\rho \\
J & =\sigma E+K,
\end{aligned}
$$

where $E$ and $H$ are the electric and magnetic field intensities, $\rho$ is the density of electrical charge, $J$ is the total current density, $\sigma E$ is the conduction current density, $B$ is the magnetic induction field, $D$ is the displacement current, $K$ is a known current density maintained by an external energy source and $\sigma$ is the conductivity.

The problem studied in this paper is suggested by geophysical modelling in which the following assumptions are made:

(1) $D=\varepsilon E, B=\mu H$ where, $\mu$ is the premeability, assumed constant and $\varepsilon$ is the permittivity,

${ }^{1}$ Department of Pure Mathematics, The University of Adelaide, Adelaide 5001, South Australia. (C) Copyright Australian Mathematical Society 1990, Serial-fee code 0334-2700/90 
(2) $\sigma$ and $\varepsilon$ are independent of the time variable,

(3) $K$ is switched on at $t=0$,

(4) for $t<0, E(\cdot, t)=0, H(\cdot, t)=0, \rho(\cdot, t)=0$.

It is then easy to see that $E$ is determined by the solution to the equation

$$
\mu \varepsilon \partial_{t}^{2} E+\mu \sigma \partial_{t} E+\nabla \times \nabla \times E=-\mu \partial_{t} K .
$$

Now formaiiy (i) may be Lapiace transformed to

$$
\left(\mu \varepsilon s^{2}+\mu \sigma s+\nabla \times \nabla \times\right) e=-\mu s k,
$$

(where $\mathscr{L}(E)=e, \mathscr{L}(K)=k$, and $s$ is the Laplace transform variable) or Fourier transformed to

$$
\left(-\mu \varepsilon \omega^{2}-\mu \sigma i \omega+\nabla \times \nabla \times\right) \tilde{e}=-i \omega \mu \tilde{k},
$$

(where $\mathscr{F}(E)=\tilde{e}, \mathscr{F}(K)=\tilde{k}$, and $\omega$ is the Fourier transform variable). In this form existence, uniqueness and regularity of the solutions was established in [1]. In particular the case $\varepsilon=0$ (the quasi-static approximation) was included and convergence of the solution as $\varepsilon \rightarrow 0$, established.

The methods of [1] are not adequate to deal with the problem considered here and for the reader's convenience we summarise the reasons. Firstly recall that the quasi-static approximation corresponds to the physical situation where the time at which observations are taken is long after the electromagnetic wave-fronts have left the observation point or, where the source is of low-frequency. These two viewpoints are equivalent to asserting that the terms $-\mu \varepsilon \omega^{2}$ and $\mu \varepsilon s^{2}$ are negligible, so that the solution to (1) is approximated in a sense made precise in [1] by the inverse transforms of the solutions to

$$
(\mu \sigma s+\nabla \times \nabla \times) e=-s \mu k
$$

or

$$
(-\mu \sigma i \omega+\nabla \times \nabla \times) \tilde{e}=-i \omega \mu \tilde{k} .
$$

Now the results in [1] assume that $\sigma>0$, whereas in the geophysical context it is often convenient to allow regions with zero conductivity (in particular to consider the case where $\sigma$ vanishes in a half-space). We note in passing that the reason the solutions of (4) and (5) are not smooth even if $K$ is smooth arises from the fact that it is usual in these applications to approximate the conductive medium (for example the earth) by making $\sigma$ piecewise constant. Then $e$ inherits this non-regularity precisely on the 'interfaces' i.e. boundaries between regions of constant $\sigma$. Nevertheless $e$ is still in $L^{2}\left(R^{3}\right)$ and the results of [1] follow from the bound:

$$
\|e\| \leq 2 \frac{1}{|s|}\left(\varepsilon_{*} \mathscr{R} s+\sigma_{*}\right)^{-1}\|k\|
$$


where

$$
\begin{gathered}
\sigma_{*}=\inf _{x \in R^{3}} \sigma(\underline{x}), \varepsilon_{*}=\inf _{x \in R^{3}} \varepsilon(\underline{x}), \\
\mathscr{R} s=\text { real part of } s(\text { and } \mathcal{F} s=\text { imaginary part of } s \text { ) }
\end{gathered}
$$

and $\|\cdot\|$ is the norm in $L^{2}\left(R^{3}\right)$. This bound is well-behaved, for a model in which $\sigma_{*}>0$, as $\varepsilon \rightarrow 0$ and it is therefore possible to take the quasi-static limit.

It should by now be clear that when $\varepsilon=0$ and $\sigma=0$ in some region this whole strategy breaks down since (6) is inapplicable.

An alternative strategy is suggested in Hohmann $[5,6]$ who considered the geophysical model of an infinite flat-earth where,

$$
\sigma(\underline{x})=\sigma(x, y, z)= \begin{cases}0, & z>0 \\ \sigma_{\text {ground }}, & z \leq 0,\end{cases}
$$

where $\sigma_{\text {ground }}>0$ and may be written as $\sigma_{\text {ground }}=\sigma_{\text {host }}+\left(\sigma_{\text {body }}-\sigma_{\text {host }}\right) \chi_{V}$ with $\sigma_{\text {body }}$ and $\sigma_{\text {host }}$ constant and $\chi_{V}$ the characteristic function of a bounded subset $V$ of $R^{3}$. Thus one is modelling the situation of a buried ore body of finite volume $V$.

From the vector diffusion equation, which is obtained from (1) by making the quasistatic approximation, viz.:

$$
\mu \sigma \partial_{t} E+\nabla \times \nabla \times E=-\mu \partial_{t} K .
$$

Hohmann derives an integral equation which is asserted to be equivalent to the vector diffusion equation in the region where $\sigma$ is non-vanishing:

$$
E=G * K+G *\left(\sigma_{V} \chi_{V}\right) E,
$$

where $\sigma_{V}$ is the difference $\sigma_{\text {host }}-\sigma_{\text {body }}, G$ is the pointwise limit, as $\varepsilon$ vanishes, of the kernel of the Greens' operator for the transmission problem with a source in the region $z \leq 0$ in the presence of an insulating upper half-space with $\varepsilon$ non-zero and $*$ denotes a modified convolution operator whose meaning is made clear in the appendix.

The integral equation approach currently forms the basis of a variety of numerical methods of solving Maxwell's equations in the geophysical modelling context [5, 6 and references therein] and hence it is of some interest to justify mathematically the approximations made in this method.

An innovative alternative approach has been proposed by Joly [7] (and developed extensively of him and co-workers) using a discretisation scheme of Nedelec [10]. Joly's technique is to replace equation (9) in the region $z>0$ by an integral boundary operator (incorporating any source in this region) on a certain Hilbert space of functions on the interface. This is matched with the solution for $z<0$ using the electromagnetic boundary conditions. The 
electric field in the region $z<0$ is never calculated, one computes only the electric field in the region $z<0$ and on the interface. This is sufficient to model the usual geophysical situations but does not address the issues considered here of how well the solution of the degenerate system (where $\sigma$ vanishes for $z>0$ ) approximates the solution of the original Maxwell's equations.

We remark that there is an extensive geophysical literature on numerical schemes for solving Maxwell's equations some of which may traced from [5, $6,12]$. To our knowledge, with the exception of Joly's work, little is known about the numerical analysis of these methods.

Our results may be summarised as follows:

(i) In Section 2.2 we give a variational form of the problem and in Sections 2.3 to 2.5 , a direct proof of the existence and uniqueness of solutions to the vector and scalar diffusion equations (without recourse to Laplace transforms) using a modification of arguments in Treves [16, pp. 397-405] in the case where $\sigma_{*}>0$.

(ii) Concurrently we find that if we allow $\sigma=0$ in a half-space, the argument in (i) still works for the scalar equation with the modification that the solution lies in a weighted $L^{2}$ space, however, the existence proof breaks down in the vector case. Thus while one can easily solve degenerate parabolic equations of the form

$$
\sigma \dot{u}-\nabla^{2} u=g
$$

the sense in which

$$
\sigma \underline{\dot{u}}+\nabla \times \nabla \times \underline{u}=\underline{g}
$$

is solvable is a much more subtle problem. (Note that the results of this section were obtained independently in the preprint in [7] while the technique used in [7] has the additional merit that it also handles the degenerate case of (10).)

As remarked above our approach is motivated by $[5,6]$ and so:

(iii) In Section 3.1 we establish an equivalence between Hohmann's integral equation and the variational form of the problem used in Section 2 in the case $\sigma_{*}$ is non-vanishing. Denoting by $G_{\sigma_{\text {arr }}}$ the kernel of the fundamental solution of (8) in the case where $\sigma$ has the general form (7), except that $\sigma(\underline{x})$ is a constant $\left(=\sigma_{\mathrm{air}} \neq 0\right)$ for $z>0$, it was observed in [9] that $G_{\sigma_{\mathrm{aur}}} \rightarrow G$ pointwise in the region $z<0$ as $\sigma_{\text {air }} \rightarrow 0$. This suggests studying the operator from $L^{2}\left([0, T] ;\left(L^{2}(V)\right)^{3}\right)$ to $L^{2}\left([0, T] ;\left(L^{2}\left(R_{-}^{3}\right)\right)^{3}\right)$ given by $F \mapsto G * \sigma_{V} \chi_{V} F$. We sketch in the appendix how to show, using the explicit expression for $G$ given there, that this operator is bounded. It follows that for small $\sigma_{V}$ a solution to the integral equation exists even when $\sigma_{\text {air }}=0$. This then gives a solution to the differential equation (8)in the region $z \leq 0$ and $\sigma_{\text {air }}=0$. 
(iv) In Section 3.3 we consider the question of how well this solution to the degenerate problem approximates the solution of the original Maxwell equations. Using [1] it is sufficient to investigate the convergence of the solutions of (8) as $\sigma_{\text {air }} \rightarrow 0$. Because we use properties of the explicit form of the fundamental solution we have to work with the Laplace transformed problem. We establish weak convergence (in $L^{2}\left(R_{-}^{3}\right)$ ) of the solution to (8) as $\sigma_{\text {air }} \rightarrow 0$ to the solution of the integral equation with $\sigma_{\text {air }}=0$.

(v) In Section 3.2 we examine briefly the boundary conditions at the interface indicating how the results in [1] may be used to establish regularity even when $\sigma_{\text {air }}=0$.

As we have remarked, the arguments in (iii) depend on [9] and so for the reader's convenience we give in the appendix the explicit form of the kernel $G$, its Laplace transform and sketch the relevant discussion from [9]. We depend on [15] for some formulae used in the weak convergence argument and the reader will need to refer to that to fill in some details. We also consider (in the appendix) the decay of the electric field in the region where $\sigma_{\text {air }}=0$ (checking the so-called radiation condition). We conclude the appendix with some remarks on general existence results for the degenerate problem.

The notation is standard (see [8]): $W^{j}(\Omega)=W^{j, 2}(\Omega)$ is the Sobolev space of functions in $L^{2}(\Omega), \Omega \subset R^{3}$, with distributional derivatives of order less than or equal to $j$ in $L^{2}(\Omega)$. An asterisk denotes convolution, $\partial_{l}$ denotes partial differentiation with respect to $l,\langle\cdot, \cdot\rangle_{H}$ is the inner product in the Hilbert space $H$, where $H$ may be $L^{2}(\Omega),\left(L^{2}(\Omega)\right)^{3}$, etc., depending upon the context and $D(A)$ denotes the domain of the operator $A$ on $H$.

\section{Existence and uniqueness of the solution of the generalised diffusion equation}

\subsection{Introduction}

In this section the existence and uniqueness of solutions to the vector and scalar diffusion equations are investigated. We will denote by $\mathscr{\theta}$, depending on context, either the operator $-\nabla^{2}$ or the operator $\nabla \times \nabla \times$. That is, we study the equation $\sigma \dot{u}+\mathscr{O} u=g$. Note that $\sigma$ must be bounded below by a positive constant in the case $\mathscr{\theta}=\nabla \times \nabla \times$ since the method we use to prove the existence and uniqueness of the solution fails when $\sigma$ vanishes in a halfspace. When $\mathscr{\theta}=-\nabla^{2}$ it is possible to allow $\sigma$ to vanish in a half-space, thus solving a degenerate parabolic/elliptic problem. 


\subsection{The generalised diffusion equation}

The vector/scalar generalised diffusion equation is

$$
\sigma \dot{u}(\underline{x}, t)+\mathscr{O} u(\underline{x}, t)=g(\underline{x}, t)
$$

with initial condition $u(\underline{x}, 0)=0$, for all $\underline{x} \in R^{3}$ where

$$
\begin{gathered}
g \in L^{2}\left(\hat{K}^{3} \times\left[\hat{0}, i j ; R^{n}\right),\right. \\
\sigma(\underline{x})= \begin{cases}\sigma_{\mathrm{air}}, & \underline{x} \in R_{+}^{3} \\
\sigma_{\operatorname{ground}(\underline{x})}, & \underline{x} \in R_{-}^{3},\end{cases}
\end{gathered}
$$

where $\sigma_{\text {air }}$ is a non-negative constant, $\sigma_{\text {ground }}(\underline{x})$ is an arbitrary positive, bounded function with lower bound $\sigma_{*}>0$, the integer $n$ denotes 1 when $\mathscr{\theta}$ is scalar and 3 otherwise, $\underline{x}=(x, y, z)$ and $T$ is a finite, non-negative real number. Note that all functions are real-valued and that all spaces are over the reals. Firstly, we replace $u$ by $\exp (-\tau t) u$, where $\tau$ is a positive real number. (We will eventually restrict $\tau$ to be greater than some $\tau_{0} \geq 0$.) In the case $\sigma_{\text {air }} \neq 0$ this substitution ensures that we obtain a contraction semi-group. (We note in passing that semigroup methods are applicable to this problem only when $\sigma_{\text {air }} \neq 0$.) Thus, upon making the above substitution, (11) becomes

$$
\sigma \dot{u}+\tau \sigma u+\mathscr{O} u=\exp (-\tau t) g=\Gamma \text {. }
$$

We define

$$
B_{\mathscr{O}}(u, v)=\left\langle\mathscr{O}^{1 / 2} u, \mathscr{O}^{1 / 2} v\right\rangle_{L^{2}\left(R^{3}\right)} .
$$

where $\langle\cdot, \cdot\rangle_{L^{2}\left(R^{3}\right)}$ indicates the inner product in $\left(L^{2}\left(R^{3}\right)\right)^{n}$. Note that $\mathscr{O}^{1 / 2}$ exists since the operators we are dealing with are positive, self-adjoint and closed (For $-\nabla^{2}$ this is a standard result. For $\nabla \times \nabla \times$ see [1].) For the cases of interest to us:

$$
\begin{aligned}
& B_{-\nabla^{2}}(u, v)=\sum_{i=1}^{3}\left\langle\partial_{x_{i}} u, \partial_{x_{i}} v\right\rangle_{L^{2}\left(R^{3}\right)}, \\
& B_{\nabla \times \nabla \times}(u, v)=\sum_{i=1}^{3}\left\langle\partial_{x_{i}}(1-Q) u, \partial_{x_{i}}(1-Q) v\right\rangle_{L^{2}\left(R^{3}\right)},
\end{aligned}
$$

where $Q$ is the pseudo-differential operator defined by

$$
\nabla \times \nabla \times=-\nabla^{2}(1-Q) \text {. }
$$

That is, loosely speaking

$$
Q_{i j}=\partial_{x_{i}} \partial_{x_{j}} / \nabla^{2}
$$


We now define the spaces $\Phi_{\ell}$ and $E_{\ell}$ in which we seek the solution to (11):

$\Phi_{\mathscr{Q}}$ is the completion of $\left(C_{c}^{\infty}\left(R^{3}\right)\right)^{n}$ with respect to the topology induced by the norm corresponding to the following inner product:

$$
\langle u, v\rangle_{\Phi_{\mathcal{Q}}}=\langle\sigma u, v\rangle_{L^{2}\left(R^{3}\right)}+B_{\mathcal{O}}(u, v) .
$$

The reason our argument fails when $\mathscr{O}=\nabla \times \nabla \times$ and $\sigma_{\text {air }}=0$ is that a function $u$ which has its support in $R_{+}^{3}$ and is longitudinal, (i.e. $\nabla \times$ $u=0$ ), must satisfy $\|u\|_{\Phi_{\nabla \times \nabla \times}}=0$, i.e. the inner product merely induces a semi-norm. Hence we exclude this case from our consideration. Note however that using Schowalter [14] we can produce an existence result even for this case by quotienting out the subspace in which (12) is degenerate. This is not particularly useful in the geophysical context unless there is an embedding of the quotient space into a function space for it is only then that the electromagnetic boundary conditions can be formulated. Unfortunately we have not found such an embedding.

$E_{\Theta}$ is defined as the completion of $L^{2}\left([0, t] ;\left(C_{c}^{\infty}\left(R^{3}\right)\right)^{3}\right)$ in the norm

$$
\|u\|_{E_{\theta}}=\sqrt{\int_{0}^{T}\|u\|_{\Phi_{\theta}}^{2}+\left\|\sigma \frac{d u}{d t}\right\|_{\Phi_{\theta}^{\prime}}^{2} d t .}
$$

(If $H$ is a Banach space, $H^{\prime}$ denotes its dual). That is,

$$
E_{\mathscr{O}}=\left\{u \in L^{2}\left([0, T] ; \Phi_{\mathscr{O}}\right) \mid \sigma d u / d t \in L^{2}\left([0, T] ; \Phi_{\mathscr{O}}^{\prime}\right)\right\} .
$$

We now define the bilinear form on $E_{\Theta} \times E_{\mathcal{\theta}}$ corresponding to the weak form of (12):

$$
A_{(\tau, \mathcal{O})}(u, v)=\int_{0}^{T}-\langle u, \sigma \dot{v}\rangle d t+\int_{0}^{T}\langle\tau \sigma u, v\rangle_{L^{2}\left(R^{3}\right)} d t+\int_{0}^{T} B_{\mathcal{O}}(u, v) d t,
$$

where the notation $\langle\cdot, \cdot\rangle$ without a subscript denotes the bracket of duality. Thus the weak form of (12) is to find a $u$ such that

$$
A_{(\tau, \mathcal{Q})}(u, v)=\int_{0}^{T}\langle\Gamma, v\rangle d t
$$

for all $v \in E_{\mathscr{Q}}$. The following result shows that $\Phi_{\mathscr{Q}}$ is a space of functions:

Clalm. Let $W$ be the space of functions $u$ with

$$
\|u\|_{W}=\int_{R^{3}} \frac{u^{2}}{(1+\theta(z) z)^{2}} d x d y d z<\infty .
$$

( $\theta$ is the Heaviside function.) Then, if $\sigma_{\mathrm{air}} \neq 0$ or in the case where $\sigma_{\mathrm{air}}=0$, $\mathscr{O}=-\nabla^{2}$ (scalar), $\Phi_{\mathscr{\theta}} \subseteq W$. 
To see why this is the case we firstly note that if $\sigma_{\text {air }} \neq 0$ then $\Phi_{\mathscr{Q}} \subset$ $L^{2}\left(R^{3}\right) \subset W$, so we need only consider the case $\sigma_{\text {air }}=0, \mathscr{O}=-\nabla^{2}$ (scalar). Let $u \in C_{c}^{\infty}\left(R^{3}\right)$, then

therefore

$$
\partial_{z} \frac{u^{2}}{(1+\theta(z) z)}=\frac{-\theta(z) u^{2}}{(1+\theta(z) z)^{2}}+\frac{\partial_{z} u^{2}}{(1+\theta(z) z)}
$$

$$
\int_{-\infty}^{\infty} \frac{\theta(z) u^{2}}{(1+\theta(z) z)^{2}} d z=\int_{-\infty}^{\infty} \frac{\partial_{z} u^{2}}{(1+\theta(z) z)} d z
$$

as $u$ and thus $u^{2}$ is of compact support. Now

$$
\begin{aligned}
& \int_{R^{3}} \frac{\theta(z) u^{2}}{(1+\theta(z) z)^{2}} d x d y d z=\int_{R^{3}} \frac{\partial_{z} u^{2}}{(1+\theta(z) z)} d x d y d z \\
& \quad \leq 2\left(\int_{R^{3}} \frac{u^{2}}{(1+\theta(z) z)^{2}} d x d y d z\right)^{1 / 2}\left(\int_{R^{3}}\left(\partial_{z} u\right)^{2} d x d y d z\right)^{1 / 2}
\end{aligned}
$$

that is,

$$
\int_{R_{+}^{3}} \frac{u^{2}}{(1+\theta(z) z)^{2}} d x d y d z \leq\|u\|_{W}\|\nabla u\|_{L^{2}\left(R^{3}\right)}
$$

Let

$$
\begin{gathered}
\alpha^{2}=\int_{R_{+}^{3}} \frac{u^{2}}{(1+\theta(z) z)^{2}} d x d y d z, \\
\beta^{2}=\int_{R_{-}^{3}} u^{2} d x d y d z, \gamma=\|\nabla u\|_{L^{2}\left(R^{3}\right)},
\end{gathered}
$$

then (17) implies

$$
\alpha^{2} \leq 2\left(\alpha^{2}+\beta^{2}\right)^{1 / 2} \gamma
$$

This forces

$$
\begin{aligned}
\alpha^{2} & \leq 2 \gamma^{2}\left(1+\sqrt{\beta^{2}+\gamma^{2}}\right) \\
& \leq 2 \gamma^{2}+2 \sqrt{\beta^{4}+2 \beta^{2} \gamma^{2}+\gamma^{4}}=4 \gamma^{2}+2 \beta^{2},
\end{aligned}
$$

and thus

$$
\alpha^{2}+\beta^{2} \leq 4\left(\beta^{2}+\gamma^{2}\right) .
$$

Now, we define $\sigma_{*}$ as inf ${\underline{x} \in R_{-}^{3}} \sigma(\underline{x})$. Thus, if $\sigma_{*} \leq 1$, then

and if $\sigma_{*}>1$

$$
\beta^{2}+\gamma^{2} \leq \frac{1}{\sigma_{*}}\left(\sigma_{*} \beta^{2}+\gamma^{2}\right)
$$

$$
\beta^{2}+\gamma^{2} \leq \sigma_{*} \beta^{2}+\gamma^{2}
$$

Noting that $\|\cdot\|_{\Phi_{\mathcal{O}}}$ is equivalent, when $\sigma_{\mathrm{air}}=0$, to $\sqrt{\sigma_{*}\|\cdot\|_{L^{2}\left(R_{-}^{3}\right)}^{2}+B_{\mathcal{Q}}(\cdot, \cdot)}$ it can be seen that there is a constant $K_{\sigma}$ such that

$$
\|u\|_{W} \leq K_{\sigma}\|u\|_{\Phi_{\theta}}
$$

that is, $\Phi_{\mathscr{O}} \subseteq W$. 
When $\mathscr{O}=\nabla \times \nabla \times$ and $\sigma_{\text {air }}$ is zero we cannot obtain an analogue of (21) and hence the method described here for existence fails. (Note that (21) also appears in the preprint in [7].)

We now discuss the dual of $\Phi_{\vartheta}$.

For $\theta=-\nabla^{2}$ and $\sigma_{\text {air }} \neq 0$ we have that $\Phi_{\mathscr{\theta}}$ is just the Sobolev space $\left(W^{1,2}\left(R^{3}\right)\right)^{n}$ and therefore the dual of $\Phi_{\mathcal{O}}$ is merely $\left(W^{-1,2}\left(R^{3}\right)\right)^{n}$. However, if $\sigma_{\text {air }}=0$, the situation is more complicated. In this case, $\sigma \dot{v}$ has support only in the lower half-space and therefore we are interested in the duality between $\Phi_{\theta}$ and $\Phi_{\theta}^{\prime}$ only for the subspace of $\Phi_{\theta}$ consisting of functions with support in $R_{-}^{3}$. However, this subspace is $\left(W^{1,2}\left(R_{-}^{3}\right)\right)^{n}$ and hence its dual is $\left(W^{-1,2}\left(R_{-}^{3}\right)\right)^{n}$. Thus, the space $E_{\Theta}$ is the space of functions $v$ such that

(1) $v \in L^{2}\left([0, T] ; \Phi_{\mathscr{Q}}\right)$

(2) $\sigma \dot{v} \in L^{2}\left([0, T] ;\left(W^{-1,2}\left(R_{-}^{3}\right)\right)^{n}\right)$.

For $\theta=\nabla \times \nabla \times$ the situation is slightly more complicated. Since $\Phi_{\theta} \subset$ $\left(L^{2}\left(R^{3}\right)\right)^{3}$, if $\sigma_{\text {air }} \neq 0$, we can resolve $u \in \Phi_{\mathcal{Q}}$ into its transverse and longitudinal components which we will denote by $u_{\mathscr{G}}$ and $u_{\mathscr{L}}$ respectively. Note that

$$
\begin{aligned}
& \left\|u_{\mathscr{I}}\right\|_{\Phi_{0}}^{2}=\left\langle\sigma u_{\mathscr{T}}, u_{\mathscr{T}}\right\rangle_{L^{2}\left(R^{3}\right)}+\left\langle\nabla u_{\mathscr{T}}, \nabla u_{\mathscr{T}}\right\rangle_{L^{2}\left(R^{3}\right)} \\
& \left\|u_{\mathscr{L}}\right\|_{\Phi_{0}}^{2}=\left\langle\sigma u_{\mathscr{L}}, u_{\mathscr{L}}\right\rangle_{L^{2}\left(R^{3}\right)} .
\end{aligned}
$$

Now for $\sigma_{\text {air }} \neq 0$, (22) implies that $u_{\mathscr{G}} \in\left(W^{1,2}\left(R^{3}\right)\right)^{3}$ and $u_{\mathscr{L}} \in\left(L^{2}\left(R^{3}\right)\right)^{3}$. That is, $\Phi_{\nabla \times \nabla \times}=\left(W_{\mathscr{T}}^{1,2}\left(R^{3}\right)\right)^{3} \oplus\left(L_{\mathscr{L}}^{2}\left(R^{3}\right)\right)^{3}(\mathscr{T}, \mathscr{L}$ indicate the transverse and longitudinal subspaces respectively) and therefore

$$
\Phi_{\nabla \times \nabla \times}^{\prime}=\left(\left(W_{\mathscr{G}}^{1,2}\left(R^{3}\right)\right)^{3}\right)^{\prime} \oplus\left(\left(L_{\mathscr{L}}^{2}\left(R^{3}\right)\right)^{3}\right)^{\prime} .
$$

It is also of interest to consider in exactly which space the solution to (11) lies. Now when $\mathscr{O}=\nabla \times \nabla \times,(11)$ is

$$
\sigma \dot{u}(\underline{x}, t)+\nabla \times \nabla \times u(\underline{x}, t)=g(\underline{x}, t)
$$

where $g \in L^{2}\left(R^{3} \times[0, T]\right)$. (We ignore initial conditions for the moment and consider the case $\theta=\nabla \times \nabla \times, \sigma_{\text {air }}=0$, even though we have no existence proof in this case.) Now, for (11) to be meaningful it is necessary that $\nabla \times u$ and $\nabla \times \nabla \times u$ be elements of $\left(L^{2}\left(R^{3}\right)\right)^{3}$. That is,

$$
u_{g} \in \begin{cases}\left(W^{1,2}\left(R^{3}\right)\right)^{3}, & \sigma_{\text {air }} \neq 0 \\ \left(W^{1,2}\left(R_{-}^{3}\right)\right)^{3}, & \sigma_{\text {air }}=0\end{cases}
$$

which implies that

$$
\nabla \times \nabla \times u_{\mathscr{T}}=-\nabla^{2} u_{\mathscr{T}} \in \begin{cases}\left(W^{-1,2}\left(R^{3}\right)\right)^{3}, & \sigma_{\text {air }} \neq 0 \\ \left(W^{-1,2}\left(R_{-}^{3}\right)\right)^{3}, & \sigma_{\text {air }}=0 .\end{cases}
$$


This, in view of (11), implies that

$$
\sigma \dot{u}_{\mathscr{T}} \in \begin{cases}\left(W^{-1,2}\left(R^{3}\right)\right)^{3}, & \sigma_{\mathrm{air}} \neq 0 \\ \left(W^{-1,2}\left(R_{-}^{3}\right)\right)^{3}, & \sigma_{\mathrm{air}}=0 .\end{cases}
$$

We also have from (11) that

$$
\sigma \dot{u}_{\mathscr{L}} \in \begin{cases}\left(L^{2}\left(R^{3}\right)\right)^{3}, & \sigma_{\mathrm{air}} \neq 0 \\ \left(L^{2}\left(R_{-}^{3}\right)\right)^{3}, & \sigma_{\text {air }}=0 .\end{cases}
$$

That is

$$
u \in\left(W_{\mathscr{T}}^{1,2}(\Omega)\right)^{3} \oplus\left(L_{\mathscr{L}}^{2}(\Omega)\right)^{3} \& \sigma \dot{u} \in\left(\left(W_{\mathscr{G}}^{1,2}(\Omega)\right)^{3}\right)^{\prime} \oplus\left(\left(L_{\mathscr{L}}^{2}(\Omega)\right)^{3}\right)^{\prime},
$$

where $\Omega=R_{-}^{3}\left(R^{3}\right)$ when $\sigma_{\text {air }}=0(\neq 0)$.

By a similar argument it can be seen that for $\mathscr{\theta}=-\nabla^{2}$ the solution $u \in$ $\left(W^{1,2}(\Omega)\right)^{n}$ and that $\sigma \dot{u} \in\left(W^{-1,2}(\Omega)\right)^{n}$.

\subsection{Existence of solution}

We now prove the existence of a solution to the generalised diffusion equation. To do this we consider a space of the form $\mathscr{G} \times \mathscr{J}$ where $E_{\mathscr{O}} \subseteq \mathscr{G}$ and $\mathscr{I}$ contains the function corresponding to our choice of initial condition. A subset of $\mathscr{G} \times \mathscr{J}$ is chosen so that:

(1) the pair $\left(v, v_{0}\right)$ corresponds to a choice of a function $v \in E_{\mathscr{O}}$ with $\lim _{t \rightarrow 0}\left\|v-v_{0}\right\|_{\mathcal{I}}=0$

(2) $\left\|\left(v, v_{0}\right)\right\| \mathscr{F}_{\times \mathcal{I}} \leq\left|A_{(\tau, \mathcal{O})}(v, v)\right|$.

Lions' generalisation of the Lax-Milgram lemma is then used to prove that there exists a unique solution to the weak differential equation which satisfies the initial conditions.

Firstly we define $\mathscr{I}$ to be the completion of $\left(C_{c}^{\infty}\left(R^{3}\right)\right)^{n}$ with respect to the norm induced by the inner product $\langle\sigma u, v\rangle_{L^{2}\left(R^{3}\right)}$. We take $\mathscr{G}=L^{2}\left([0, T] ; \Phi_{\mathcal{O}}\right)$ and thus $\mathscr{G} \times \mathscr{I}=M=L^{2}\left([0, T] ; \Phi_{\Theta}\right) \times \mathscr{I}$ with norm

$$
\left\|\left(u, u_{0}\right)\right\|_{M}=\sqrt{\int_{0}^{T}\|u\|_{\Phi_{\Theta}}^{2} d t+\left\langle\sigma u_{0}, u_{0}\right\rangle_{L^{2}\left(R^{3}\right)}}
$$

We choose as the subset of $M, \hat{h} \subset M$ as

$$
\left\{\left(v, v_{0}\right) \in M \mid v \in E_{\mathcal{\theta}}, \lim _{t \rightarrow T}\|v\|_{\mathscr{I}}=0 \& \lim _{t \rightarrow 0}\left\|v-v_{0}\right\|_{\mathscr{I}}=0\right\} .
$$

If $\sigma_{\text {air }}=0$ we make the additional restriction upon $\mathscr{I}$ that its element have support in $R_{-}^{3}$, i.e. it is the completion of $\left(C_{c}^{\infty}\left(R_{-}^{3}\right)\right)^{n}$ with respect to $\langle\sigma \cdot, \cdot\rangle_{L^{2}\left(R^{3}\right)}$. Now,

$$
2 \int_{0}^{T}\langle\sigma \dot{u}, u\rangle d t=\left\langle\sigma u_{T}, u_{T}\right\rangle_{L^{2}\left(R^{3}\right)}-\left\langle\sigma u_{0}, u_{0}\right\rangle_{L^{2}\left(R^{3}\right)},
$$


and thus

$$
\begin{aligned}
& 2 A_{(\tau, \mathcal{O})}(u, u)+\left\langle\sigma u_{T}, u_{T}\right\rangle_{L^{2}\left(R^{3}\right)} \\
& =\left\langle\sigma u_{0}, u_{0}\right\rangle_{L^{2}\left(R^{3}\right)}+2\left(\int_{0}^{T}\left[\langle\tau \sigma u, u\rangle_{L^{2}\left(R^{3}\right)}+B_{\mathcal{O}}(u, u) d t\right]\right) .
\end{aligned}
$$

Assume without loss of generality that $\tau \geq 1$; thus we have the energy inequality:

$$
2 A_{(\tau, \mathscr{Q})}(u, u)+\left\langle\sigma u_{T}, u_{T}\right\rangle_{L^{2}\left(R^{3}\right)} \geq\left\langle\sigma u_{0}, u_{0}\right\rangle_{L^{2}\left(R^{3}\right)}+2 \int_{0}^{T}\langle\sigma u, u\rangle+B_{\mathscr{O}(u, u)} d t .
$$

Hence for $h \in \hat{h}$,

$$
\|h\|_{M}^{2} \leq\left|A_{(\tau, \mathcal{O})}(h, h)\right| .
$$

Note that $A_{(\tau, \mathcal{O})}(w, h)$ is clearly a continuous linear functional on $\boldsymbol{M}$ for every fixed $\left(h, h_{0}\right) \in \hat{h}$. We now use Lions' generalisation of the Lax-Milgram Lemma (Treves [16], p. 403).

LEMMA. Let $\mathbf{E}$ be a Hilbert space, $\tilde{h}$ a linear subspace of $\mathbf{E}, \mathbf{U}(w, h)$ a sesquilinear functional on $\mathbf{E} \times \tilde{h}$ having the following properties: E.

(a) for each fixed $h \in \tilde{h}, w \rightarrow \mathbf{U}(w, h)$ is a continuous linear functional on

(b) there is a $c_{0}>0$ such that, for every $h \in \tilde{h}, c_{0}\|h\|_{E}^{2} \leq|\mathbf{U}(h, h)|$.

Under these circumstances we have the

Conclusion. There is a bounded linear map $G$ of the antidual $\overline{\mathbf{E}}^{\prime}$ of $\mathbf{E}$ into $\mathbf{E}$, with norm $\leq c_{0}^{-1}$, such that for every continuous linear functional $\lambda$ on $\mathbf{E}$,

$$
\mathbf{U}(G \lambda, h)=\lambda(h), \forall h \in \tilde{h} .
$$

So let our continuous functional $\lambda$ be

$$
v \mapsto \int_{0}^{T}\langle\Gamma, v\rangle_{L^{2}\left(R^{3}\right)} d t .
$$

Choose our $\tilde{h}$ to be $\hat{h}$ and $\mathbf{E}$ to be $M$. The energy inequality for $A_{(\tau, \mathcal{Q})}$ on $\hat{h}$ and the fact that $\left(w, w_{0}\right) \mapsto A_{(\tau, \mathcal{O})}(w, h)$ is continuous on $M$ for fixed $\left(h, h_{0}\right) \in \hat{h}$, $\left(\omega, \omega_{0}\right) \in M$, shows that we can take $\mathrm{U}(u, v)=A_{\tau, \mathcal{O}}(u, v)$ and apply the lemma to obtain the

THEOREM. There exists $\left(V, V_{0}\right) \in M$ such that

$$
A_{(\tau, \mathscr{O})}(V, h)=\int_{0}^{T}\langle\Gamma, h\rangle_{L^{2}\left(R^{3}\right)} d t \forall h \in \hat{h}
$$


Choose $h \in C_{c}^{\infty}\left([0, T] ; \Phi_{\mathcal{O}}\right)(\subset \hat{h} \subset M)$ so

$$
\int_{0}^{T}\left[\langle-\sigma V, \dot{h}\rangle_{L^{2}\left(R^{3}\right)}+\langle\sigma \tau V, h\rangle_{L^{2}\left(R^{3}\right)}+B_{\mathscr{Q}}(V, h)\right] d t=\int_{0}^{T}\langle\Gamma, h\rangle_{L^{2}\left(R^{3}\right)} d t,
$$

and therefore,

$$
\sigma \dot{V}+(\tau \sigma V+\mathscr{O} V)=\Gamma
$$

in sense of $\Phi_{\theta}^{\prime}$ valued distributions on $[0, T]$.

\subsection{Initial conditions}

First we prove a technical result.

LEMMA. The natural injection $C^{\infty}\left([0, T] ; \Phi_{\mathscr{O}}\right) \rightarrow C^{0}([0, T] ; \mathscr{I})$ can be extended to a continuous map $E_{\mathscr{O}} \rightarrow C^{0}([0, T] ; \mathscr{I})$ (we equip $C^{0}([0, T] ; \mathscr{I}$ ) with the natural norm $\sup _{0<t<T}\left\|u_{t}\right\|_{\mathscr{F}}$ where $\left.u_{t_{0}}=\left.u(\underline{x}, t)\right|_{t=t_{0}}\right)$.

Proof. Let $u \in E_{\mathscr{O}}$. We define $\tilde{u}$ on $(-T, T)$ by

$$
\tilde{u}= \begin{cases}u_{t}, & t>0 \\ u_{-t}, & t<0\end{cases}
$$

It is clear that the map $u \mapsto \tilde{u}$ is a continuous injection, with norm 2 , from $E_{\mathscr{Q}}=E_{\mathscr{Q}}([0, T])$ to $E_{\mathscr{Q}}((-T, T))$. Consider now

$$
\alpha \in C^{\infty}(R), \alpha_{t}= \begin{cases}0, & t<-T \\ 1, & t>0\end{cases}
$$

Let $\left.u \in C^{\infty}([0, T]) ; \Phi_{\mathcal{Q}}\right)$. Note that when $\sigma_{\text {air }}=0$ we are really dealing with a truncation of $u$,

$$
\operatorname{Trunc}(u)(\underline{x})= \begin{cases}u(\underline{x}), & z \leq 0 \\ 0, & \text { otherwise }\end{cases}
$$

since we require the elements of $\mathscr{I}$ to have support in $R_{-}^{3}$. However, since $\langle\sigma u, u\rangle_{L^{2}\left(R^{3}\right)}=\langle\sigma \operatorname{Trunc}(u), \operatorname{Trunc}(u)\rangle_{L^{2}\left(R^{3}\right)}$ this is not a problem.

We now consider

$$
\begin{aligned}
\langle\sigma(\alpha \tilde{u}),(\alpha \tilde{u})\rangle_{L^{2}\left(R^{3}\right)} & =2 \int_{-T}^{t}\left\langle(\alpha \tilde{u})_{s}, \sigma(\alpha \dot{\tilde{u}})_{s}\right\rangle d s \\
& \leq \int_{-T}^{T}\left\|(\alpha \tilde{u})_{s}\right\|_{\Phi_{\theta}}^{2}+\left\|\sigma(\alpha \dot{\tilde{u}})_{s}\right\|_{\Phi_{\theta}^{\prime}}^{2} d s \\
& \leq C_{\alpha} \int_{-T}^{T}\left\|\tilde{u}_{s}\right\|_{\Phi_{\theta}}^{2}+\left\|\sigma \dot{\tilde{u}}_{s}\right\|_{\Phi_{\theta}^{\prime}}^{2} d s \leq 2 C_{\alpha}\|u\|_{E_{\Theta}} .
\end{aligned}
$$

Since $\alpha \tilde{u}$ restricted to $[0, T]$ equals $u$, we see that the natural injection

$$
C^{\infty}\left([0, T] ; \Phi_{\mathcal{O}}\right) \rightarrow C^{0}([0, T] ; \mathscr{I})
$$


is continuous and thus has a unique continuous extension to all of $E_{\mathscr{Q}}$, since $C^{\infty}\left([0, T] ; \Phi_{\mathcal{Q}}\right)$ is, clearly, dense in $E_{\mathscr{\theta}}$.

Now (23) implies $\sigma \dot{V} \in L^{2}\left([0, T] ; \Phi_{\mathscr{\theta}}^{\prime}\right)$ i.e. $V \in E_{\mathscr{O}}$, hence we can consider $V$ as a continuous function on $[0, T]$, with values in $\mathscr{I}$. Thus,

$$
\int_{0}^{T}\langle-\sigma V, \dot{h}\rangle_{L^{2}\left(R^{3}\right)} d t=\left.\langle\sigma V, h\rangle_{L^{2}\left(R^{3}\right)}\right|_{t=0}+\int_{0}^{T}\langle\sigma \dot{V}, h\rangle_{L^{2}\left(R^{3}\right)} d t .
$$

So by (23)

$$
\left.\langle\sigma V, h\rangle_{L^{2}\left(R^{3}\right)}\right|_{t=0}=0 \text { for all } h \in \hat{h},
$$

i.e., $\sigma V_{0}=0$ a.e. in $R^{3}$ and therefore so does $V_{0}$, since if $\sigma$ has support only in $R_{-}^{3}$ we have chosen $\mathscr{I}$ to ensure that the initial data $V_{0}$ is zero in $R_{+}^{3}$.

\subsection{Uniqueness of the solution}

If we have two solutions $u, v \in E_{\mathscr{\theta}}$ to the above problem then $W=u-v$ is an element of $E_{\mathcal{O}}$ which satisfies

$$
\sigma \dot{W}+(\tau \sigma+\mathscr{O}) W=0, \quad W_{0}=0 \quad \text { in } R^{3} .
$$

We now show, using the bilinear form $A_{(\tau, \mathcal{Q})}^{\prime}$ defined on $E_{\mathscr{Q}} \times E_{\mathcal{Q}}$ by

$$
A_{(\tau, \mathcal{O})}^{\prime}(u, v)=\int_{0}^{T}\left(\langle\sigma \dot{u}, v\rangle+\langle\tau \sigma u, v\rangle_{L^{2}\left(R^{3}\right)}+B_{\mathcal{O}}(u, v)\right) d t,
$$

that $W \equiv 0$ in $L^{2}\left([0, T] ; \Phi_{\mathcal{O}}\right)$.

Firstly, by a similar argument to that for $A_{(\tau, \mathcal{Q})}$ above we have:

$$
2 A_{(\tau, \mathcal{Q})}^{\prime}(W, W)+\left\langle\sigma W_{0}, W_{0}\right\rangle_{L^{2}\left(R^{3}\right)} \geq\left\langle\sigma W_{T}, W_{T}\right\rangle_{L^{2}\left(R^{3}\right)}+2\|W\|_{L^{2}\left([0, T] ; \Phi_{\Theta}\right)}^{2} .
$$

Now

$$
A_{(\tau, \mathcal{Q})}^{\prime}(W, W)=\int_{0}^{T}\langle\sigma \dot{W}+(\tau \sigma+\mathscr{O}) W, W\rangle d t=0
$$

and

$$
\left\langle\sigma W_{0}, W_{0}\right\rangle_{L^{2}\left(R^{3}\right)}=0
$$

by (24). Therefore

$$
0 \geq\left\langle\sigma W_{T}, W_{T}\right\rangle_{L^{2}\left(R^{3}\right)}+2\|W\|_{L^{2}\left([0, T] ; \Phi_{\theta}\right)}^{2} \geq 0,
$$

and hence $W \equiv 0$ in $L^{2}\left([0, T] ; \Phi_{\Theta}\right)$.

\section{Equivalence of the weak form of the \\ Vector diffusion equation and Hohmann's integral equation}

In this section we establish the validity of Hohmann's [5, 6] method of approximating the electric field in the case of an insulating upper half-space. 
The main problem in this case is that there is no Green's tensor since the operator $\sigma(\underline{x}) \partial_{t}+\nabla \times \nabla \times$ has a nonzero kernel. Specifically, if $u$ is a transverse infinitely differentiable function with compact support in the upper half-space then $\left(\sigma(\underline{x}) \partial_{t}+\nabla \times \nabla \times\right) u=0$. Thus, the inverse of the operator cannot exist without further restrictions. Hohmann's [6] method implicitly places a restriction on the solution, viz. that it is the limit as $\varepsilon \rightarrow 0$ in some sense of the solutions to $\left(\bar{\nabla} \times \bar{\nabla} \times+\sigma(\underline{x}) \partial_{t}+\varepsilon \hat{\partial}_{t}^{2}\right) u=\hat{f}, \sigma_{\text {air }}=\hat{0}$, which satisíy a radiation condition at infinity.

Our first result shows that the solutions to the vector diffusion equation with $\sigma_{\text {air }} \neq 0$ are bounded in $L^{2}\left(R_{-}^{3}\right)$ independently of the conductivity of the upper half-space, if the source has support in the ground.

We have from [1] the inequality:

$$
|s|\langle u, \varepsilon u\rangle_{L^{2}\left(R^{3}\right)}+\langle u, \sigma u\rangle_{L^{2}\left(R^{3}\right)} \leq 2\langle u, f\rangle_{L^{2}\left(R^{3}\right)},
$$

which implies that

$$
|s|\langle u, \sigma u\rangle_{L^{2}\left(R^{3}\right)} \leq 2\langle u, f\rangle_{L^{2}\left(R^{3}\right)} .
$$

Thus, if we define $P: L^{2}\left(R^{3}\right) \rightarrow L^{2}\left(R_{-}^{3}\right)$ by

$$
P u(\underline{x})= \begin{cases}u(\underline{x}), & \underline{x} \in R_{-}^{3} \\ 0, & \underline{x} \in R_{+}^{3}\end{cases}
$$

we have the following:

$$
|s|\langle P u, P \sigma u\rangle_{L^{2}\left(R^{3}\right)} \leq 2\langle P u, f\rangle_{L^{2}\left(R^{3}\right)} .
$$

That is

$$
\|P u\|_{L^{2}\left(R_{-}^{3}\right)} \leq \frac{2}{\sigma_{*}|s|}\|f\|_{L^{2}\left(R_{-}^{3}\right)},
$$

(where $\sigma_{*}=\inf _{x \in R_{-}^{3}} \sigma(\underline{x})$ ). Thus, there is a subsequence of the sequence of solutions, ordered by the value of the conductivity of the upper half-space, which converges weakly in $L^{2}\left(R_{-}^{3}\right)$. We will establish in Section 3.3 that this weak limit satisfies the Laplace transformed integral equation (at least for small $\sigma_{V}$ ) when $V$ is bounded.

It follows therefore that our next task should be to establish the sense in which the integral equation holds.

\subsection{Equivalence of the weak form' of the vector diffusion equation and Hohmann's integral equation}

Here we show that the weak form of the vector diffusion equation

$$
\left(\nabla \times \nabla \times+\sigma(\underline{x}) \partial_{t}\right) u=-\mu \partial_{t} K
$$

is equivalent to an integral equation involving the field $E^{\prime}(\underline{x}, t)$ generated by the source $K$ for the case where $\sigma_{V}=0$ and the fundamental solution to the above equation where $\sigma_{V}=0$. 
We will assume for that the region $V$ where $\sigma_{V} \neq 0$ is contained in $R_{-}^{3}$ and is compact. We first consider the case in which $\sigma$ is non-vanishing.

Consider $E^{\prime}(\underline{x}, t) \in L^{2}([0, T] ; D(\nabla \times \nabla \times))$ such that for

$$
K(\underline{x}, t) \in\left\{S \in C_{c}^{\infty}\left(R^{3} \times[0, T]\right) \mid \nabla \cdot S(\underline{x}, t)=0, \forall t \in[0, T]\right\}
$$

the equation

$$
\nabla \times \nabla \times E^{\prime}(\underline{x}, t)+\mu_{0} \sigma_{0} \partial_{t} E^{\prime}(\underline{x}, t)=-\mu_{0} \partial_{t} K(\underline{x}, t)
$$

with initial condition

$$
E^{\prime}(\underline{x}, t)=0 \forall t \leq 0 \text { and } \forall \underline{x} \in R^{3}
$$

holds weakly, where

$$
\sigma_{0}(\underline{x})= \begin{cases}\sigma_{+}, & \underline{x} \in R_{+}^{3} \\ \sigma_{-}, & \underline{x} \in R_{-}^{3},\end{cases}
$$

with $\sigma_{+}, \sigma_{-}$both positive constants. (That is, $E^{\prime}(\underline{x}, t)$ is the solution to the vector diffusion equation in the case $\sigma_{V}=0$.)

Let $G_{\sigma_{\mathrm{air}}}\left(\underline{x}, \underline{x}^{\prime}, t\right)$ be the solution of (in the sense of distributions)

$$
\begin{gathered}
\nabla \times \nabla \times G_{\sigma_{\mathrm{atr}}}\left(\underline{x}, \underline{x}^{\prime}, t\right)+\mu_{0} \sigma_{0} \partial_{t} G_{\sigma_{\mathrm{air}}}\left(\underline{x}, \underline{x}^{\prime}, t\right)=-\mu_{0} \partial_{t} I \delta(x, t) \\
G_{\sigma_{\mathrm{atr}}}\left(\underline{x}, \underline{x}^{\prime}, t\right)=0, \quad t \leq 0 .
\end{gathered}
$$

( $I$ is the identity tensor.) Note that $G_{\sigma_{\text {air }}}\left(\underline{x}, \underline{x}^{\prime}, t\right)$ is known explicitly in terms of its Laplace transform [15]. Hohmann [6] refers to this as the Green's dyadic when $\sigma_{\text {air }}=0$, though this is not immediately apparent because of a misprint in that paper.

Let $E \in L^{2}\left([0, T] ;\left(L^{2}\left(R^{3}\right)\right)^{3}\right)$ be the solution of

$$
E(\underline{x}, t)=E^{\prime}(\underline{x}, t)+G_{\sigma_{\text {atr }}} * \sigma_{V} \chi_{V} E(\underline{x}, t)
$$

with

$$
E(\underline{x}, t)=0, \quad t \leq 0,
$$

where $\sigma_{V}$ is some positive constant and $\chi_{V}$ is the characteristic function of $V$. The modified convolution operator $E \mapsto G_{\sigma_{\text {air }}} * \sigma_{V} \chi_{V} E$ is well defined since it is just the operator which we denoted by $G$ in section 2.3 and therefore defines a bounded operator on $L^{2}\left([0, T] ;\left(L^{2}\left(R^{3}\right)\right)^{3}\right)$. Now a solution of $(9)$ exists in $L^{2}\left([0, T] ;\left(L^{2}(V)\right)^{3}\right)$ (for small $\sigma_{V}$ ) by application of a Picard argument.

The solution is then extended to all of $R^{3}$ using (38) and the known form of $G_{\sigma_{\text {alr }}}$. Next from (38) we have

$$
\mu_{0} \sigma_{0} \partial_{t} E(\underline{x}, t)=\mu_{0} \sigma_{0} \partial_{t} E^{\prime}(\underline{x}, t)+\mu_{0} \sigma_{0} \partial_{t} G * \sigma_{V} \chi_{V} E(\underline{x}, t)
$$


weakly, i.e. in $\mathscr{D}^{\prime}\left(R^{3}\right)$. Using the properties of $G_{\sigma_{\text {alr }}}$ we calculate that the right hand side of $(40)$ equals

$$
\begin{aligned}
& -\nabla \times \nabla \times E^{\prime}(\underline{x}, t)-\mu_{0} \partial_{t} K(\underline{x}, t) \\
& +\int_{0}^{t} \int_{V}\left(-\nabla \times \nabla \times G_{\sigma_{\mathrm{arr}}}\left(\underline{x}, \underline{x}^{\prime}, t-t^{\prime}\right)-\mu_{0} \delta\left(\underline{x}-\underline{x}^{\prime}, t-t^{\prime}\right)\right) \sigma_{V} \chi_{V} E\left(\underline{x}^{\prime}, t^{\prime}\right) d x^{\prime} d t^{\prime} \\
& +\mu_{0} \sigma_{0} \int_{V} G_{\sigma_{\mathrm{arr}}}\left(\underline{x}, \underline{x}^{\prime}, 0\right) \sigma_{V} \chi_{V} E\left(\underline{x}^{\prime}, t\right) d x^{\prime} \\
& =-\nabla \times \nabla \times\left(E^{\prime}(\underline{x}, t)+\int_{0}^{t} \int_{V} G_{\sigma_{\mathrm{air}}}\left(\underline{x}, \underline{x}^{\prime}, t, t^{\prime}\right) \sigma_{V} \chi_{V} E\left(\underline{x}^{\prime}, t^{\prime}\right) d v^{\prime} d t^{\prime}\right) \\
& \quad-\mu_{0} \partial_{t} K(\underline{x}, t)-\sigma_{V} \chi_{V} \partial_{t} E(\underline{x}, t) .
\end{aligned}
$$

Therefore,

$$
\nabla \times \nabla \times E(\underline{x}, t)+\mu_{0}\left(\sigma_{0}+\sigma_{V} \chi_{V}\right) \partial_{t} E(\underline{x}, t)=-\mu_{0} \partial_{t} K(\underline{x}, t) .
$$

But $\sigma_{0}+\sigma_{V} \chi_{V}=\sigma$; thus

$$
\nabla \times \nabla \times E(\underline{x}, t)+\mu_{0} \sigma \partial_{t} E(\underline{x}, t)=-\mu_{0} \partial_{t} K(\underline{x}, t)
$$

in the weak sense. That is, $E^{\prime}(\underline{x}, t)$ is a weak solution of the vector diffusion equation.

Conversely, if $E(\underline{x}, t)$ is a weak solution of the vector diffusion equation,

$$
\nabla \times \nabla \times E(\underline{x}, t)+\mu_{0} \sigma \partial_{t} E(\underline{x}, t)=-\mu_{0} \partial_{t} K(\underline{x}, t) ;
$$

then

$$
\nabla \times \nabla \times E(\underline{x}, t)+\mu_{0} \sigma_{0} \partial_{t} E(\underline{x}, t)=-\mu_{0} \sigma_{V} \chi_{V} \partial_{t} E(\underline{x}, t)-\mu_{0} \partial_{t} K(\underline{x}, t),
$$

i.e. $E(\underline{x}, t)$ is response to source $\sigma_{V} \chi_{V} E(\underline{x}, t)+K(\underline{x}, t)$, i.e.

$$
\begin{aligned}
E(\underline{x}, t) & =G_{\sigma_{\mathrm{air}}} *\left(\sigma_{V} \chi_{V} E+J\right)(\underline{x}, t) \\
& =G_{\sigma_{\mathrm{air}}} * K(\underline{x}, t)+G_{\sigma_{\mathrm{air}}} * \sigma_{V} \chi_{V} E(\underline{x}, t) \\
& =E^{\prime}(\underline{x}, t)+G_{\sigma_{\mathrm{air}}} * \sigma_{V} \chi_{V} E(\underline{x}, t) .
\end{aligned}
$$

That is, $E(\underline{x}, t)$ satisfies the integral equation.

The case $\sigma_{\text {air }}=0$ is similar. Although we cannot show a priori that $E^{\prime}(\underline{x}, t) \in L^{2}\left([0, T] ;\left(L^{2}\left(R_{-}^{3}\right)\right)^{3}\right)$ or that there is a fundamental solution of the vector diffusion equation which is a bounded integral operator on $L^{2}\left([0, T] ;\left(L^{2}\left(R_{-}^{3}\right)\right)^{3}\right)$, it nevertheless follows from the argument in the appendix (cf. [9]) that for $\sigma_{\text {air }}=0$ the operator $F \mapsto G * \sigma_{V} \chi_{V} F$ (note $G_{\sigma_{\text {aur }}}=G$ when $\left.\sigma_{\text {air }}=0\right)$ from $L^{2}\left([0, T] ;\left(L^{2}(V)\right)^{3}\right)$ to $L^{2}\left([0, T] ;\left(L^{2}\left(R_{-}^{3}\right)\right)^{3}\right)$ is bounded. Hence for small $\sigma_{V}$ we can again use a Picard type method to get a solution to (38). Thus, considering the weak form of the vector diffusion equation in the ground (in $\mathscr{D}^{\prime}\left(R_{-}^{3}\right)$ ) the argument above from equation (39) onwards holds 
in this sense. Thus, in the case of an insulating upper half-space, the integral equation and the weak form of the vector diffusion equation are equivalent in the region $z \leq 0$. The solution in the region $z>0$ is then computed from (39) using the known form of $G\left(\underline{x} \mid \underline{x}^{\prime}\right)$ for $\underline{x}^{\prime} \in V$ and $\underline{x}$ in the upper half-space [15].

REMARK: Note that if $J$ is transverse and has no $z$-component then $E(\underline{x}, t)$ satisfies the scalar diffusion equation (0'Brien and Smith [13]). Hence by the results of Section $2, E(\underline{x}, t)$ lies in the weighted Sovolev space in that case.

\subsection{Boundary conditions}

In the quasi-static limit there are no true total surface charges. Since we assume that $D=\varepsilon E$ we have from Maxwell's equations that if $\varepsilon=0$ then

$$
\nabla \cdot D=\nabla \cdot \varepsilon E=0 .
$$

However, Hohmann's integral equation contains a perturbation term corresponding to the response to a scattering current $j_{s}$ which has associated with it a rate of change of surface charge $\nabla \cdot j_{s}=\nabla \cdot \sigma_{V} \chi_{V} E$. Now, since the surface charge is always zero, this implies that

$$
\nabla \cdot \sigma(\underline{x}) E=0
$$

and writing $\sigma(\underline{x})=\sigma_{V} \chi_{V}+\sigma_{0}$ we have that

$$
\nabla \cdot \sigma_{V} \chi_{V} E=-\nabla \cdot \sigma_{0} E,
$$

which reflects the physically obvious fact that the field in the region $z \leq$ 0 gains its longitudinal component as a consequence of the presence and geometry of the region $V$ of differing conductivity.

We now turn to the behaviour of the field at interfaces. In [1] it was shown that for $\Omega$, a region spanning the boundary $\Gamma$ between two regions $\Omega_{-}, \Omega_{+}$, that

Lemma. Suppose $f \in L^{2}(\Omega)$ and $\nabla \cdot f=0$ in $\Omega$. Let $n \cdot f_{\mp}$ denote the normal trace of $n \cdot f$ on $\Gamma$ from $\Omega_{\mp}$ then

$$
n \cdot f_{-}=n \cdot f_{+}
$$

in the sense of equality of distributions in $H^{-1 / 2}(\Gamma)$.

Now for a source in the region $z \leq 0$ the field $E$ in the case $\sigma_{\text {ground }}$ is constant is in $L^{2}\left(R_{-}^{3}\right)$ and when $\sigma_{V}$ is non-zero, the field, as given by the solution to Hohmann's integral equation, is also in $L^{2}\left(R_{-}^{3}\right)$. Thus, we have that, irrespective of the presence or absence of a region $V$ of differing conductivity, $\sigma E=0$ in the region $z>0, E \in L^{2}\left([0, T] ;\left(L^{2}\left(R_{-}^{3}\right)\right)^{3}\right)$ and $\nabla \cdot \sigma E=0$. Thus, the conditions of the lemma are satisfied and we have that the normal 
component of $\sigma E$ is continuous across all interfaces, (in the sense of traces on the interfaces). A similar discussion establishes that the other regularity results of [1] hold here as well.

\subsection{The weak convergence argument}

In this section the source $k$ is transverse (i.e. $\nabla \cdot k=0$ in an $L^{2}$ sense) and

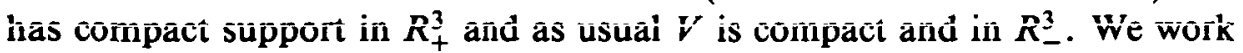
with the Laplace transformed version of (38):

$$
e(\underline{x})=e_{\sigma_{\mathrm{atr}}}^{\prime}(\underline{x})+\mu s \sigma_{V} \int_{R^{3}} G_{\sigma_{\mathrm{aur}}}\left(\underline{x} \mid \underline{x}^{\prime}\right) \chi v\left(\underline{x}^{\prime}\right) e\left(\underline{x}^{\prime}\right) d \underline{x}^{\prime}
$$

where $G_{\sigma_{\text {air }}}\left(\underline{x} \mid \underline{x}^{\prime}\right)$ is given in [15] for $\sigma_{\text {air }} \neq 0$ and for $\sigma_{\text {air }}=0$ in the appendix. We regard this as an equation to the solved in $L^{2}\left(R_{-}^{3}\right)$, that is we fix the Laplace transform variable (see however the remark below). As shown by a typical calculation in the appendix, the explicit expressions in [15] can be used to show that the operator from $L^{2}(V)$ to $L^{2}\left(R_{-}^{3}\right)$ given by $F \mapsto \hat{F}$ where

$$
\hat{F}(\underline{x})=\int_{R_{-}^{3}} G_{\sigma_{\text {air }}}\left(\underline{x} \mid \underline{x}^{\prime}\right) \chi_{V}\left(\underline{x}^{\prime}\right) F\left(\underline{x}^{\prime}\right) d \underline{x}^{\prime}
$$

converges in the weak operator topology as the parameter $\sigma_{\text {air }}$ goes to zero to the corresponding operator for $\sigma_{\text {air }}=0$ (given by integration against $G_{0}\left(\underline{x} \mid \underline{x}^{\prime}\right)=G\left(\underline{x} \mid \underline{x}^{\prime}\right)$ ). (To establish this in the time domain in the absence of an explicit form for the fundamental solution when $\sigma_{\text {air }} \neq 0$ requires considerably more work and hence we restrict our discussion to the Laplace transform version.)

From this we deduce

(i) The equation (47) has a solution in $L^{2}(V)$ for $\sigma_{V}$ sufficiently small by the contraction mapping theorem. This solution may then be extended to all of $R_{-}^{3}$ using (47).

(ii) The solution to (47) is the norm limit as $n \rightarrow \infty$ of the sequence $\left(e_{n}^{\sigma_{\text {arr }}}\right)$ defined iteratively by

$$
e_{n+1}^{\sigma_{\mathrm{air}}}(\underline{x})=e_{\sigma_{\mathrm{air}}}^{\prime}(\underline{x})+\int_{R^{3}} G_{\sigma_{\mathrm{aur}}}\left(\underline{x} \mid \underline{x}^{\prime}\right) \chi \chi_{V}\left(\underline{x}^{\prime}\right) e_{n}^{\sigma_{\mathrm{air}}} d \underline{x}^{\prime}
$$

(iii) We also note in the appendix that the same argument which establishes the weak convergence of the operator in (19) as $\sigma_{\text {air }}$ goes to zero also suffices to show that $e_{\sigma_{\text {ar }}}^{\prime}$ converges weakly to $e_{0}^{\prime}$ in $L^{2}\left(R_{-}^{3}\right)$ as $\sigma_{\text {air }} \rightarrow 0$ provided the source $k$ is in $L^{2}\left(R_{+}^{3}\right)$ with compact support. Putting these facts together we deduce the main result of this section.

(iv) The solution $e_{n}^{\sigma_{\mathrm{ar}}}$ of (49) converges weakly as $\sigma_{\mathrm{air}} \rightarrow 0$ to the solution $e$ of

$$
e(\underline{x})=e_{0}^{\prime}(\underline{x})+\mu s \sigma_{V} \int_{R^{3}} G\left(\underline{x} \mid \underline{x}^{\prime}\right) e\left(\underline{x}^{\prime}\right) d \underline{x}^{\prime}
$$


in $L^{2}\left(R_{-}^{3}\right)$ for sources which are transverse and of compact support in $R_{-}^{3}$.

REMARKS. A more detailed inspection of the convergence arguments in the appendix reveals that the convergence as $\sigma_{\text {air }} \rightarrow 0$ is actually uniform in the Laplace transform variable and so the inverse Laplace transform may be taken. The details are somewhat lengthy so we omit this refinement. We also note that the preceding argument works when $\sigma_{V} \chi_{V}$ is replaced by any $L^{\infty}$ function of sufficiently small norm with support in $V$.

\subsection{Conclusion}

We have established an existence and uniqueness result for an approximate form of Maxwell's equations used in geophysical modelling. These equations are degenerate parabolic-elliptic diffusion equations. The case of the degenerate vector equation defies standard methods and so existence in this case is established using an integral equation introduced by Hohmann. That this integral equation has solutions is proved using the explicit from of the Green's tensor for the half-space problem. We have also shown that we can approximate the Laplace transform of the solution of the degenerate problem weakly in the ground by the solution of the Laplace transformed non-degenerate equation. Finally we note that we cannot establish this approximation directly in the time domain because we have insufficient information on $G_{\sigma_{\text {air }}}$. However one can nevertheless establish boundedness of the $L^{2}$ norm of the solution to (9) independently of $\sigma_{\text {air }}$.

\section{Acknowledgement}

We thank Professor P. Joly for bringing the preprint in [7] to our attention and for some helpful remarks.

\section{Appendix}

The first part of this appendix summarises the estimates which allow the argument in Section 3.3 to go through. Because these estimates use the explicit form of the solution of

$$
\nabla \times \nabla \times G\left(\underline{x} \mid \underline{x}^{\prime}\right)+s \sigma \mu_{0} G\left(\underline{x} \mid \underline{x}^{\prime}\right)=-\mu_{0} s \delta\left(\underline{x}-\underline{x}^{\prime}\right) I,
$$

we cannot give the full details and still keep this paper to a reasonable length. Consequently we sketch the main ideas using for illustration the explicit form of the solution to this equation only in the case $\sigma_{\mathrm{air}}=0$. To avoid confusion with the literature we will change our conventions at this point and henceforth the subset of $R^{3}$ with $z \geq 0$ has conductivity $\sigma_{\text {ground }}$ ( $=\mathrm{a}$ constant) and the 
complement has conductivity $\sigma_{\mathrm{air}}$. Then the so called Green's tensor for this problem when $\sigma_{\text {air }}=0$ is the solution to the preceding equation, which we write in the form $G\left(\underline{x} \mid \underline{x}^{\prime}\right)=G_{p}\left(\underline{x} \mid \underline{x}^{\prime}\right)+G_{s}\left(\underline{x} \mid \underline{x}^{\prime}\right)$, where

$$
\begin{aligned}
G_{p}\left(\underline{x} \mid \underline{x}^{\prime}\right)= & \frac{k_{2}^{2}}{\sigma}\left(\begin{array}{ccc}
\alpha_{1} & 0 & \\
0 & \alpha_{1} & 0 \\
0 & 0 & \alpha_{1}
\end{array}\right) \\
& +\frac{1}{\sigma}\left(\begin{array}{ccc}
\partial_{x} \partial_{x^{\prime}} \alpha_{1} & \partial_{x} \partial_{y^{\prime}} \alpha_{1} & \partial_{x} \partial_{z} \alpha_{1} \\
\partial_{y} \partial_{x^{\prime}} \alpha_{1} & \partial_{y} \partial_{y^{\prime}} \alpha_{1} & \partial_{y} \partial_{z^{\prime}} \alpha_{1} \\
\partial_{z} \partial_{x^{\prime}} \alpha_{1} & \partial_{z} \partial_{y^{\prime}} \alpha_{1} & \partial_{z^{\prime}} \partial_{z} \alpha_{1}
\end{array}\right)
\end{aligned}
$$

and

$$
\begin{aligned}
G_{s}\left(\underline{x} \mid \underline{x}^{\prime}\right)= & \frac{1}{\sigma}\left(\begin{array}{ccc}
\alpha_{3} & 0 & 0 \\
0 & \alpha_{3} & 0 \\
0 & 0 & -\alpha_{2}
\end{array}\right) \\
& +\frac{k_{2}^{2}}{\sigma}\left(\begin{array}{ccc}
\partial_{x} \partial_{x^{\prime}} \alpha_{4} & \partial_{x} \partial_{y^{\prime}} \alpha_{4} & \partial_{x} \partial_{z^{\prime}} \alpha_{2} \\
\partial_{y} \partial_{x^{\prime}} \alpha_{4} & \partial_{y} \partial_{y^{\prime}} \alpha_{4} & \partial_{y} \partial_{z^{\prime}} \alpha_{2} \\
\partial_{z} \partial_{x^{\prime}} \alpha_{2} & \partial_{z} \partial_{y^{\prime}} \alpha_{2} & \partial_{z^{\prime}} \partial_{z} \alpha_{2}
\end{array}\right),
\end{aligned}
$$

where,

$$
\begin{aligned}
\sigma & =\sigma_{\text {ground }}, k_{2}=-i \sqrt{\mu \sigma s} \\
\alpha_{1} & =\frac{1}{4 \pi} \frac{\exp \left(-i k_{2} R\right)}{R} \\
\alpha_{2} & =\frac{1}{4 \pi} \frac{\exp \left(-i k_{2} R_{2}\right)}{R_{s}} \\
\alpha_{3} & =\frac{1}{4 \pi} \int_{0}^{\infty}\left(\frac{K-\lambda}{K+\lambda}\right) \frac{\lambda}{K} J_{0}(\lambda \rho) \exp \left(-K\left(z+z^{\prime}\right)\right) d \lambda \\
\alpha_{4} & =\frac{1}{4 \pi} \int_{0}^{\infty}\left(2-\frac{\lambda}{K}\right) J_{0}(\lambda \rho) \exp \left(-K\left(z+z^{\prime}\right)\right) d \lambda \\
K^{2} & =\lambda^{2}+\mu \sigma_{\text {ground }} s, \rho=\sqrt{\left(x-x^{\prime}\right)^{2}+\left(y-y^{\prime}\right)^{2}}
\end{aligned}
$$

with $R=\left|\underline{x}-\underline{x}^{\prime}\right|$ and $R_{s}$ obtained by replacing $z^{\prime}$ by $-z^{\prime}$ in $R$ and $z \geq z^{\prime} \geq 0$. For the case $z^{\prime} \geq z \geq 0$ we merely interchange $z$ and $z^{\prime}$ in the above formulae. The action of $G$ on $J \in\left(L^{2}\left(R_{-}^{3}\right)\right)^{3}$ is given by integration with respect to the primed variables. Note that this is the modified convolution referred to in the introduction where the $z^{\prime}$ variable appears in the $G_{s}$ terms in the combination $z+z^{\prime}$ not $z-z^{\prime}$.

In the general case the solution $G_{\sigma_{\text {air }}}$ of

$$
\nabla \times \nabla \times G_{\sigma_{\text {ant }}}\left(\underline{x} \mid \underline{x}^{\prime}\right)+s \sigma \mu_{0} G_{\sigma_{\text {aIr }}}\left(\underline{x} \mid \underline{x}^{\prime}\right)=-\mu_{0} s \delta\left(\underline{x}-\underline{x}^{\prime}\right) I
$$

for $\underline{x}, \underline{x}^{\prime} \in R^{3}$ is given in [15]. One notes that $G_{\sigma_{\text {air }}}$ can be written as the sum

$$
G_{\sigma_{\mathrm{air}}}=G_{p}\left(\underline{x} \mid \underline{x}^{\prime}\right)+G_{s}^{\prime}\left(\underline{x} \mid \underline{x}^{\prime}\right)
$$


where $G_{p}\left(\underline{x} \mid \underline{x}^{\prime}\right)$ is given by (1) and $G_{s}^{\prime}\left(\underline{x} \mid \underline{x}^{\prime}\right)$ has the same form as (52) except that $\alpha_{2}, \alpha_{3}, \alpha_{4}$ are slightly modified. As (51) is the solution to the vector diffusion equation when the conductivity is constarit $\left(=\sigma_{\text {ground }}\right)$ and hence is well understood it suffices to consider only the operator defined by integration against $G_{s}^{\prime}\left(\underline{x} \mid \underline{x}^{\prime}\right)$. We will consider in detail just the case where $\sigma_{\text {air }}=0$ as the modifications to the Green's tensor introduced by allowing $\sigma_{\text {air }} \neq 0$ can be seen from [15], to, at worst, only modify the succeeding estimates by inessential constants. The method of proof is to establish for $\mathscr{R} s>0$ an inequality of the form

$$
\left|\left(\rho^{2}+\left(z+z^{\prime}\right)^{2}\right) G_{s}\left(\underline{x} \mid \underline{x}^{\prime}\right)\right|^{2} \leq \text { constant. }
$$

We illustrate the argument by considering just the term $\left(\rho^{2}+\left(z+z^{\prime}\right)^{2}\right) \alpha_{3}$. (The other terms are handled in a similar way). Now

$$
\left|\left(z+z^{\prime}\right)^{2} \alpha_{3}\right| \leq\left(z+z^{\prime}\right)^{2} \text { (const) } \int_{0}^{\infty} \lambda \exp -\lambda\left(z+z^{\prime}\right) d \lambda<\text { constant } .
$$

The proof that $\left|\rho^{2} \alpha_{3}\right|$ is bounded uses some manipulations with Bessel function recurrence relations to write

$$
\rho^{2} \frac{\lambda}{K} J_{0}(\lambda \rho) \frac{K-\lambda}{K+\lambda} \exp -K\left(z+z^{\prime}\right)
$$

in the form

$$
J_{0}(\lambda \rho) F_{1}\left(\lambda, z+z^{\prime}\right)+J_{1}(\lambda \rho) F_{2}\left(\lambda, z+z^{\prime}\right)
$$

where $F_{j}$ are functions whose integral with respect to $\lambda$ is bounded independently of $z$ as above.

This means that given any pair $u, v$ of functions in $L^{2}\left(R_{-}^{3}\right)$ the function on $R_{-}^{3} \times R^{3}$ given by

$$
u(\underline{x}) \chi_{v}\left(\underline{x}^{\prime}\right) G_{s}\left(\underline{x} \mid \underline{x}^{\prime}\right) v\left(\underline{x}^{\prime}\right)
$$

is bounded in modulus by a constant times the function

$$
\left|u(\underline{x}) \chi_{v}\left(\underline{x}^{\prime}\right)\left(\rho^{2}+\left(z+z^{\prime}\right)^{2}\right)^{-1} v\left(\underline{x}^{\prime}\right)\right|
$$

independently of the value of $\sigma_{\text {air }}$.

Using the analogues of (53) (see [9] or [15]) for the case $\sigma_{\mathrm{air}} \neq 0$ one sees that $G_{\sigma_{\text {anr }}}\left(\underline{x} \mid \underline{x}^{\prime}\right)$ converges pointwise to $G\left(\underline{x} \mid \underline{x}^{\prime}\right)$. Hence the observation of the previous paragraph enables us to use the dominated convergence theorem to deduce convergence (in the weak operator topology on the bounded operators from $L^{2}(V)$ to $L^{2}\left(R_{-}^{3}\right)$ ) of the operator of integration against $G_{\sigma_{\mathrm{air}}}\left(\underline{x} \mid \underline{x}^{\prime}\right) \chi_{V}\left(\underline{x}^{\prime}\right)$ to the operator of integration against $G\left(\underline{x} \mid \underline{x}^{\prime}\right) \chi_{V}\left(\underline{x}^{\prime}\right)$.

We remark that one can prove the same fact (again using the explicit formulae in [15]) when $V$ is allowed to lie in the region with $z<0$. The argument is essentially identical. 


\section{A.1 Inverse Laplace transform of the Green's dyadic}

We can now find the time-domain version of the elements of the tensor. We follow Hohmann [5].

First we note that (Oberhettinger and Badii [11])

$$
\begin{aligned}
\mathscr{S}^{-1}\left(\frac{\exp \left(-k_{2} R_{s}\right)}{R_{s}}\right) & =\mathscr{S}^{-1}\left(\frac{\exp \left(-\sqrt{(s \mu \sigma)} R_{s}\right)}{R_{s}}\right) \\
& =\frac{\sqrt{\mu \sigma}}{2 \sqrt{\pi} t^{3 / 2}} \exp \left(-\frac{\mu \sigma R_{s}^{2}}{4 t}\right),
\end{aligned}
$$

where $\sigma=\sigma_{\text {ground. }}$ Secondly,

$$
\mathscr{L}^{-1}\left(s^{-1 / 2} \exp \left(-\sqrt{s \mu \sigma} R_{s}\right)\right)=\frac{1}{\sqrt{\pi t}} \exp \left(-\frac{\mu \sigma R_{s}^{2}}{4 t}\right) .
$$

We now verify Hohmann's [6] time domain expressions, using our notation. We first consider:

$$
\begin{aligned}
\mathscr{L}^{-1} & \left(\frac{1}{\rho} \frac{\partial \alpha_{4}}{\partial \rho}\right) \\
= & \mathscr{L}^{-1}\left(\frac{1}{4 \pi \sigma \rho} \int_{0}^{\infty}\left(2-\frac{\lambda}{K}\right) \exp \left(-K\left(z+z^{\prime}\right)\right) J_{1}(\lambda \rho) \lambda d \lambda\right) \\
= & \frac{1}{4 \pi^{3 / 2} \sigma} \frac{\left(z+z^{\prime}\right)}{r} \theta^{3} W\left(z+z^{\prime}\right) \int_{0}^{\infty} \beta \exp \left(\frac{-\beta^{2}}{4}\right) J_{1}(\beta r) d \beta \\
& -\frac{1}{2 \pi^{3 / 2} \sigma} \theta^{3} W\left(R_{s}\right) .
\end{aligned}
$$

We now define as in Hohmann [6]:

$$
\theta=\sqrt{\frac{\mu \sigma}{4 t}}, W\left(R_{s}\right)=\frac{\exp \left(-\theta^{2} R_{s}^{2}\right)}{t}, \quad \beta=\frac{\lambda}{\theta}, r=\theta \rho .
$$

We next consider

$$
\begin{aligned}
\mathscr{L}^{-1}( & \left.\frac{k_{2}^{2}}{\sigma} \alpha_{3}\right) \\
= & \mathscr{L}^{-1}\left(\frac{k_{2}^{2}}{4 \pi \sigma} \int_{0}^{\infty} \frac{K-\lambda}{K+\lambda} \frac{\lambda}{K} J_{0}(\lambda \rho) \exp \left(-K\left(z+z^{\prime}\right)\right) d \lambda\right) \\
= & -\frac{1}{2 \pi^{3 / 2} \sigma} \frac{1}{4} W\left(z+z^{\prime}\right) \theta^{3} \int_{0}^{\infty} \beta^{3} \exp \left(-\frac{\beta^{2}}{4}\right) J_{0}\left(\beta_{r}\right) d \lambda \\
& +\frac{1}{2 \pi^{3 / 2} \sigma} \theta^{3} W\left(R_{s}\right)\left(1-2\left(z+z^{\prime}\right)^{2} \theta^{2}\right) \\
& +\frac{1}{2 \pi^{3 / 2} \sigma} W\left(z+z^{\prime}\right)\left(z+z^{\prime}\right) \theta^{4} \int_{0}^{\infty} \beta^{2} \exp \left(-\frac{\beta^{2}}{4}\right) J_{0}(\beta r) d \lambda
\end{aligned}
$$


We now consider

$$
\mathscr{L}^{-1}\left(\frac{k_{2}^{2}}{4 \pi \sigma} \frac{\exp \left(-i k_{2} R_{s}\right)}{R_{s}}\right)=-\frac{\mu}{4 \pi^{3 / 2}} \partial_{i} \theta W\left(R_{s}\right) .
$$

Finally, we consider

$$
\begin{aligned}
\mathscr{L}^{-1}\left(\frac{1}{R_{s}} \frac{\partial \alpha_{2}}{\partial R_{s}}\right) & =\mathscr{L}^{-1}\left(\frac{1}{4 \pi \sigma}\left(i k_{2} R_{s}+1\right) \frac{\exp \left(-i k_{2} R_{s}\right)}{R_{s}^{3}}\right) \\
& =\frac{1}{2 \pi^{3 / 2} \sigma} \theta^{3} W\left(R_{s}\right) .
\end{aligned}
$$

We now briefly outline why the operator defined by the above kernel is bounded. Firstly, we recall that the Green's tensor in the ground, when the source is in the ground, can be written as: $G=G_{p}+G_{s}$ where $G_{p}$ is the free space Green's function for the equation

$$
\left(\nabla \times \nabla \times+\sigma \partial_{l}\right) G_{p}=-\mu \partial_{t} \delta \quad \text { (with } \sigma=\text { constant). }
$$

Note that $G_{p}$ a priori defines a bounded operator on $L^{2}\left(R^{3}\right)$.

Hence we need to show that the operator $F \mapsto G_{s} * \sigma_{V} \chi_{V} F$ is a bounded operator from $L^{2}\left([0, T] ;\left(L^{2}(V)\right)^{3}\right)$ to $L^{2}\left([0, T] ;\left(L^{2}\left(R_{-}^{3}\right)\right)^{3}\right)$. Observe that we can assume there is a real $l$ with $0<l \leq z^{\prime}$ so that the scattering terms depend upon $R_{s}$ which is bounded below by $l$ for $\underline{x}^{\prime}$ in $V$. This allows us to show that the scattering terms are either exponentially damped with respect to $R_{s}$ or are of the form $f(z) g(x, y)$ where $f \in L^{2}(R), g \in L^{2}\left(R^{2}\right)$. It can then be easily shown that $F \mapsto G_{s} * \chi_{V} F$ is bounded.

\section{A.2 The asymptotic $z$-behaviour of the solution}

It is worth checking the radiation condition in Hohmann's method as $z \rightarrow-\infty$ in the air. In can be seen from the explicit expressions for the Green's tensor that the asymptotic $z$-behaviour of the tensor depends upon the behaviour of terms of the form:

$$
\frac{1}{4 \pi} \int_{0}^{\infty} \frac{\lambda^{n}}{K \lambda} \partial^{\beta} J_{1}(\lambda \rho) \exp \left(i\left(h_{2} z^{\prime}-h_{1} z\right)\right) d \lambda .
$$

where $\rho=\left|\underline{x}-\underline{x^{\prime}}\right|$.

Using the estimates $\left|\partial_{x_{1}} \partial_{x_{1}} J_{1}(\lambda \rho)\right| \leq F(\rho) \lambda^{2}$, where $F(x)$ is a bounded continuous function, and $\left|\partial_{x_{1}} J_{1}(\lambda \rho)\right| \leq \lambda$ it can be shown that terms of the above form are dominated by $F(\rho)|s||z|^{-2}$.

Thus, as a matrix $\left\|G\left(s, \underline{x}, \underline{x}^{\prime}\right)\right\| \leq|s| 3 F(\rho)|z|^{-2}$. This estimate can be used to show that:

LEMMA. Let $F(s, x, y, z) \in L^{2}\left(R_{-}^{3}\right)$ with compact support, then $G * F \rightarrow 0$ as $z \rightarrow-\infty$.

It then follows from a theorem of Treves [16], pp. 420-421 we have $\mathscr{L}^{-1}(G * F) \rightarrow 0$ as $z \rightarrow-\infty$. 


\section{A.3 Conjecture on a general existence theorem}

From a mathematical viewpoint it may be desirable to have a proof of existence which avoids the integral equation. It is observed in [9] that such a proof cannot be formulated along the lines of this paper as, at best, the Green's function for the problem can only be defined as an operator from the space of transverse $L^{2}$ functions into a weighted Sobolev space consisting

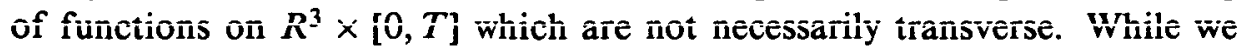
conjecture that this is true a proof would require a form of the Lax-Milgram lemma not known to us. The alternative approach of Joly [7] also indicates that this is true.

\section{References}

[1] A. L. Carey and D. M. O'Brien, "Existence and regularity results for Maxwell's equations in the quasi-static limit", J. Austral. Math. Soc. B 27 (1986) 402-415.

[2] D. H. S. Cheng, “On the formulation of the dyadic Green's function in a layered medium", Electromagnetics 6 (1986) 171-182.

[3] R. E. Colins, "Dyadic Green's function expansions in spherical co-ordinates", Electromagnetics 6 (1986) 183-207.

[4] H. Gradshteyn and G. Ryzhik, Table of integrals, series, and products (Academic Press, New York, 1987).

[5] G. W. Hohmann, "Three-dimensional induced polarisation and electromagnetic modeling", Geophysics 40 (1975) 309-324.

[6] G. W. Hohman and W. A. San Filipo, "Integral equation solution for the transient electromagnetic response of a three-dimensional body in a conductive half-space", Geophysics 50 (1985) 798-809.

[7] P. Joly, "Propagation of an electromagnetic field in a conductive half-space", (Boundary Elements 7, Eds. Brebbia, G. A. and Maier, G., Springer-Verlag, Berlin, pp. 3-53-3-68, 1985) and Y. Goldman, P. Joly, M. Kern, "The electric field in a conductive half space as a model for petroleum and mining prospection" to appear in Math. Meth. in Applied Sciences and references therein.

[8] J. L. Lions and E. Magnes, Non-homogeneous boundary value problems and applications, Vol. 2, (Springer-Verlag, Berlin, 1972).

[9] K. McNamara, M. Sc. Thesis, University of Adelaide, 1988.

[10] J. C. Nedelec, "Mixed finite elements in $R^{3 ”}$, Numerische Mathematik 35, (1980) 315-341.

[11] F. Oberhettinger and L. Badii, Tables of Laplace transforms (Springer-Verlag, 1973).

[12] D. M. O'Brien and R. S. Smith, "Transient electromagnetic response of a layered conductin medium at asymptotically late times", J. Austral. Math. Soc. B 27 (1985) 1-30.

[13] F. W. J. Olver, Asymptotics and special functions (Academic Press, New York, 1974).

[14] R. E. Showalter, "Initial and final value problems for degenerate parabolic systems", Indiana Univ. Math. Journal, 28 (1979) 883-894.

[15] Chen-To Tai, Dyadic Green's functions in electromagnetic theory (Intext, Scranton, Pennsylvania, 1971).

[16] F. Treves, Basic linear partial differential equations (Academic Press, New York, 1975).

[17] J. Van Bladel, Electromagnetic fields (New York, McGraw-Hill, 1964). 